CRS occurred in all pts (53\% CRS ${ }^{\circ} 1,29 \%{ }^{\circ} 2$ and $18 \%$ 3 ) with a median onset on day 2 (range days 0-7) and a median duration of 4 days (range 1-21). Tocilizumab was administered at least once in all pts. Ten pts (59\%) experienced Immune Effector Cell associated Neurotoxicity Syndrome (ICANS, $30 \%{ }^{\circ} 1,10 \%{ }^{\circ} 2,30 \%{ }^{\circ} 3,20 \%{ }^{\circ} 4$ and $10 \%$ $\left.{ }^{\circ} 5\right)$ with a median onset between day 7 and 8 and a median duration of 8 days (range 3-49). Cytopenia was significant following CAR T-cell treatment: all but one pts had neutropenia $<500 / \mu$ f for more than seven days.

Response assessment four weeks after CAR T-cell transfusion was available for 15 pts.

Objective response rate (ORR) at this early follow-up was $67 \%$, with complete remission (CR) in four (27\%) and partial remission (PR) in six pts (40\%). Interestingly, ORR was higher in the four pts not receiving bridging chemotherapy between leukapheresis and CAR T-cell therapy than in pts in which bridging was applied (100\% vs. 55\%). Responders had significantly higher LDH levels at apheresis, start of lymphodepletion and CAR T-cell transfusion than non-responders.

Conclusions Since January 2019, the CAR T cell program has been successfully initiated at the LMU Munich, and $17 \mathrm{r} / \mathrm{r}$ DLBCL pts have been treated at our center to date. CAR T cells induced responses in heavily pretreated pts with response rates within the expected range. Toxicity was significant but manageable in most pts. Involvement of a multidisciplinary ImmunoTaskforce was a key element for adequate patient care. Preliminary data supports the hypothesis that low tumor dynamics are associated with favorable outcomes of CD19 CAR T cell therapy.

Disclosure Information V. Bücklein: None. V. Blumenberg: None. C. Schmidt: None. K. Rejeski: None. M. Ruzicka: None. N. Müller: None. A. Reischer: None. L. von Baumgarten: None. A. Völkl: None. B. Wagner: None. A. Humpe: None. J. Tischer: None. H. Stemmler: None. M. von Bergwelt: None. M. Subklewe: None.

\section{P07.02 HIGH-AFFINITY TCRS SPECIFIC FOR CANCER TESTIS ANTIGENS AS A THERAPY FOR MULTIPLE MYELOMA AND SOLID TUMORS}

MAJ de Rooij ${ }^{*}$, DM van der Steen, D Remst, A Wouters, M van der Meent, RS Hagedoorn, MGD Kester, PA van Veelen, FJH Falkenburg, MHM Heemskerk. LUMC, Leiden, Netherlands

\subsection{6/jitc-2020-ITOC7.96}

Background Cancer Testis Antigens (CTAs) are highly expressed in multiple different tumor types, but silent in normal tissue, except the testis. This tumor-restricted expression pattern makes them an ideal target for adoptive T-cell therapy. However, the responsiveness in clinical setting may be hampered because high-affinity $\mathrm{T}$ cells against self-antigens presented in the context of self-HLA are deleted in the thymus by negative selection. In this study, we aim to identify highaffinity $\mathrm{T}$ cell receptors (TCRs) specific for CTAs from the allogeneic-HLA repertoire.

Materials and Methods In this study, HLA class I binding peptides derived from different CTA genes were identified by HLA-peptide elution experiments and subsequent mass spectrometric analysis. From the identified peptides HLA tetramers were generated to isolate peptide specific $\mathrm{CD}^{+} \mathrm{T}$ cells from healthy allogeneic donors. Efficacy and safety of the TCRs was determined by various different stimulation assays. The most potent TCRs were sequenced, analyzed and transduced into peripheral $\mathrm{CD}^{+}$and $\mathrm{CD} 4^{+} \mathrm{T}$ cells to confirm CTA specific cytokine production and cytotoxicity.

Results MAGE and CTAG peptides were eluted from multiple myelomas, EBV-transformed lymphoblastic cells, acute myeloid leukemia and ovarium carcinomas. We selected TCRs recognizing 3 different MAGE-A1 peptides in the context of HLA$A * 02: 01$, HLA-A*03:01 and HLA-B*07:02. Furthermore, we selected TCRs specific for MAGE-A3 in the context of HLA$B * 35: 01$ and HLA-A*01:01; TCRs specific for MAGE-A9 in the context of HLA-A*01:01 and TCRs specific for CTAG1 in the context of HLA-A*02:01. The selected T-cell clones demonstrated efficient recognition of MAGE-A1, MAGE-A3 or CTAG1 positive multiple myeloma and solid tumor cell lines without detectable cross-reactivity.

Conclusions We identified multiple different TCRs from the allogeneic-HLA repertoire specific for CTA genes. These TCRs demonstrate efficient recognition and killing of CTA positive multiple myeloma and solid tumor cell lines and did not show any cross-reactivity. The peptides recognized by the TCRs are presented in different HLA alleles. Since, $71 \%$ of the world population contains one of these HLA-alleles, a large percentage suffering from a MAGE or CTAG positive tumor could potentially be treated with the identified TCRs by TCR-gene therapy.

Disclosure Information M.A.J. de Rooij: None. D.M. van der Steen: None. D. Remst: None. A. Wouters: None. M. van der Meent: None. R.S. Hagedoorn: None. M.G.D. Kester: None. P.A. van Veelen: None. F.J.H. Falkenburg: None. M.H.M. Heemskerk: None.

\section{P08 Combination Therapy}

\section{P08.01 LOW-DOSE CHECKPOINT INHIBITORS WITH HYPERTHERMIA AND IL-2 ARE SAFE AND EFFECTIVE IN STAGE IV CANCER WITH UNFAVORABLE IMMUNOLOGICAL PROFILE (MSILW, PD-L1 UNDER $1 \%$, TMB ${ }^{\text {LOW }}$ - A SINGLE-INSTITUTION EXPERIENCE FROM 2015 TO 2020}

${ }^{1} \mathrm{R}$ Kleef*, ${ }^{1} \mathrm{R}$ Nagy, ${ }^{1} \mathrm{~V}$ Bacher, ${ }^{2} \mathrm{~T}$ Bakacs, ${ }^{1} \mathrm{~T}$ Lausch, ${ }^{3} \mathrm{D}$ McKee, ${ }^{4} \mathrm{R}$ Moss, ${ }^{5} \mathrm{H}$ Bojar, ${ }^{6} \mathrm{~N}$ Thoennissen. ${ }^{1}$ Ralf Kleef Immunology and Integrative Oncology, Vienna, Austria; ${ }^{2}$ PRET Therapeutics Ltd., Budapest, Hungary; Integrative Cancer Therapies, London, UK; ${ }^{4}$ Moss Reports, Blue Hill, ME, USA; ${ }^{5}$ NextGen Oncology Group, Duesseldorf, Germany; ${ }^{6}$ Oncology at Lenbachplatz, Munich, Germany

\subsection{6/jitc-2020-ITOC7.97}

Background Close to 10 million cancer deaths occurred worldwide in 2017 primarily due to stage IV disease, the management of which is still palliative by intent. Differently from melanoma and non-small cell lung cancer, where the use of ground-breaking immune checkpoint inhibitors (ICI) results in a relatively high efficacy, the response rate in many other stage IV tumors, such as gastrointestinal cancers, breast cancers, sarcomas, and part of genitourinary cancers remains low. In addition, administration of this type of cancer immunotherapy is known for its potentially severe and even fatal side effects due to their severe immune-related adverse events (irAEs).

Materials and Methods Here, we report a retrospective analysis of 129 patients with stage IV cancer who exhausted conventional treatments, who were treated by an low-dose ipilimu$\mathrm{mab}(0.3 \mathrm{mg} / \mathrm{kg})$ plus nivolumab $(0.5 \mathrm{mg} / \mathrm{kg})$ blockade in 
combination with individually dose adapted interleukin 2 (IL2) treatment under taurolidine protection and locoregionaland whole body hyperthermia.

Results The overall response (OR) rate of the 129 stage IV patients was $49.6 \%$ with an objective response (ORR) of $31.8 \%$. In 15 stage I-III cancer patients, the overall response (OR) rate and objective response (ORR) were 93\%, respectively. The entire treatment was performed as outpatient therapy which was mostly associated with a toxicity of grade 1-2 (24.4 and 14.9\%, respectively), including nausea, diarrhea, skin rash and pruritus, and elevation of liver transaminases during the first 24 hours. Only 4,76\% of the patients developed grade III and 1,79\% grade IV irAEs, such as autoimmune hepatitis, thyroid problems, acute kidney injury and/or diabetes mellitus. There were no signs of late adverse events from this treatment with follow-up greater than 5 years post therapy.

Conclusions In comparison to the commonly known rates of response and side effects in ICI, we were able to show relatively high response rates in parallel with low toxicity profile by the aid of immune response modifiers.

Disclosure Information R. Kleef: None. R. Nagy: None. V. Bacher: None. T. Bakacs: None. T. Lausch: None. D. McKee: None. R. Moss: None. H. Bojar: None. N. Thoennissen: None.

\section{P08.02 BERBERINE-LOADED LIPOSOME FORMULATION ENHANCE THE PHAGOCYTIC ACTIVITY OF LIPOSOMAL IMIQUIMOD TOWARDS COLON CANCER CELL LINES}

M Mianowska*, M Zaremba-Czogalla, A Zygmunt, J Gubernator. Wroclaw University, Wroclaw, Poland

\subsection{6/jitc-2020-ITOC7.98}

Background Colorectal cancer is the third most commonly diagnosed malignant tumor, taking fourth place in terms of cause of cancer deaths worldwide. ${ }^{1}$ Unfortunately, the ability of the immune system to distinguish its own from foreign cells is often limited. One of the overexpressed receptors is receptor CD47 - widely distributed glycoprotein on the cell surface of various kind of tumors. It plays a role as 'don't eat me' signal by binding with receptor SIRP $\alpha$, presents on the cell surface of macrophages. ${ }^{2}$ Calreticulin, protein occurring on the surface of tumor cells and phagocytes, acts as protein with pro-phagocytic properties. Several natural bioactive substances are predicted to induce immunogenic cell death by translocation calreticulin on the surface of cancer cells which significantly increases the efficiency of their phagocytosis. Moreover, one of the well-known TLR-7 receptor agonists - imiquimod, is involved in phosphorylation of Bruton's tyrosine kinase leading to the appearance of calreticulin on the surface of macrophages, which increases the efficiency of phagocytosis of tumor cells. ${ }^{3}$ Combination therapy composed of berberine and imiquimod can be highlighted as effective immunotherapy for colon cancer. However, such an approach remains very limited. Liposomes can serve as promising carriers for targeting delivery and controlled release of anti-cancer agents.

Material and Methods Liposomes were prepared by the thinfilm hydration method followed by extrusion. Human colon cancer cell line (LS180 I SW620) and human monocytic cell line (THP-1) were used for experiments. Calreticulin was detected by using confocal microscopy.
Results The work presented aimed to develop novel liposomal formulations of berberine and imiquimod which were examined for their efficacy in combination against colorectal cancer cell lines. Liposomal formulations of both compounds were successfully prepared using active loading method with different $\mathrm{pH}$ generating agents. All loading methods showed desired characteristics in terms of mean liposome size and polydispersity. The encapsulation efficiency was higher than $95 \%$ for almost all used formulations. The in vitro study proved cytotoxicity of berberine loaded liposomal formulations on tested colon cancer cell lines. The results of the immunofluorescence staining indicated that the both compounds triggered calreticulin on the cell surface (colon cancer or macrophages).

Conclusions The combination of both substances in the liposomal form may generate a synergistic effect on phagocytosis of colon cancer cells.

\section{REFERENCES}

1. Arnold M, Sierra MS, Laversanne M, et al. Global patterns and trends in colorectal cancer incidence and mortality. Gut 2017;66:683-691.

2. Sick E, Jeanne A, Schneider C, Dedieu S, Takeda K, Martiny L. CD47 update: a multifaceted actor in the tumor microenvironment of potential therapeutic interest, Br J Pharmacol 2012, 167(7):1415-30.

3. M. Feng, et al., Macrophages eat cancer cells using their own calreticulin as a guide: Roles of TLR and Btk. PNAS 2015;112( 7):2145-2150.

Disclosure Information M. Mianowska: B. Research Grant (principal investigator, collaborator or consultant and pending grants as well as grants already received); Significant; National Science Center, Poland. M. Zaremba-Czogalla: None. A. Zygmunt: None. J. Gubernator: None.

\section{P08.03 COMBINING PD-1/PD-L1 BLOCKADE AND RANKL INHIBITORS TO TREAT BREAST CANCERS UNRESPONSIVE TO STANDARD THERAPY}

C Pilard*, P Roncarati, E Hendrick, A Lebeau, D Bruyère, T Lerho, M Ancion, C Reynders, P Delvenne, M Herfs, P Hubert. Laboratory of Experimental Pathology, GIGA-Cancer, University of Liege, Liège, Belgium

\subsection{6/jitc-2020-ITOC7.99}

Background In the past decade, immunotherapy using immune checkpoint inhibitors (especially targeting the PD-1/PD-L1 axis) has been demonstrated as a promising strategy for the treatment of cancers that do not respond to classical chemoradiotherapy. Given that cancer cells have the potential to express many immunosuppressive molecules other than PD-L1, the combination of immune checkpoint inhibitors with other drugs thwarting tumor immunosuppressive microenvironment could represent a promising strategy. Among these immunosuppressive molecules, RANKL, a member of the TNF superfamily, which mainly affects the immune system and bone remodeling, has been shown to be a key factor promoting the progression of breast cancer. In addition, RANKL induces the formation of tolerogenic dendritic cells and Treg cells, which promotes immunotolerance to the tumor.

The aim of this research project is to study the impact of several RANKL inhibitors on triple negative breast cancer and to analyze the efficiency of their association with anti-PD-1/ PD-L1 agents.

Materials and Methods We studied RANKL and PD-L1 expression in several murine and human breast cancer cell lines by immunohistochemistry. The secretion of RANKL was analyzed 